\title{
Assessment of Undiscovered Continuous Oil and Gas Resources in the Wolfcamp Shale and Bone Spring Formation of the Delaware Basin, Permian Basin Province, New Mexico and Texas, 2018
}

Using a geology-based assessment methodology, the U.S. Geological Survey assessed undiscovered, technically recoverable continuous mean resources of 46.3 billion barrels of oil and 281 trillion cubic feet of gas in the Wolfcamp shale and Bone Spring Formation of the Delaware Basin in the Permian Basin Province, southeast New Mexico and west Texas.

\section{Introduction}

In 2018, the U.S. Geological Survey (USGS) completed a geology-based assessment of undiscovered, technically recoverable continuous oil and gas resources in the PennsylvanianPermian Wolfcamp shale (informal name; age follows Hamlin and Baumgardner, 2012) and the early Permian Bone Spring Formation of the Delaware Basin in the Permian Basin Province, southeast New Mexico and west Texas (figs. 1-3). The Delaware Basin is the western subbasin of the Permian Basin Province and is separated from the eastern Midland Basin by the uplifted Central Basin Platform. The Delaware Basin is rimmed by carbonate platforms, including the Central Basin and Diablo Platforms and the Northwest Shelf. The Wolfcamp shale was deposited throughout the Permian Basin and consists of interbedded, organic-rich shales and carbonates in both the Midland and Delaware Basins; however, the Wolfcamp shale in the Delaware Basin is thicker, deeper, and more thermally mature than in the Midland Basin. The overlying Bone Spring consists of alternating sandstone, carbonate, and shale cycles and is time-equivalent to the Spraberry Formation in the Midland Basin.

Recent USGS assessments of continuous oil and gas resources in the Permian Basin Province include the Wolfcamp shale (Gaswirth and others, 2016) and the Spraberry Formation (Marra and others, 2017) in the Midland Basin. The Bone Spring Formation was assessed for conventional oil and gas resources in 2007 (Schenk and others, 2008).

This is the first USGS assessment of continuous resources in the Wolfcamp shale and Bone Spring Formation of the Delaware Basin. Both the Wolfcamp shale and the Bone Spring Formation are subdivided into stratigraphic units based on petrophysical log signatures and landing zones for horizontal wells. This assessment used data from the Enerdeq ${ }^{\mathrm{TM}}$ and ProdFit ${ }^{\mathrm{TM}}$ databases (IHS Markit ${ }^{\circledR}$, $2018 \mathrm{a}, \mathrm{b}$ ) for information on well landing zones, well production, and unit depths and thicknesses.

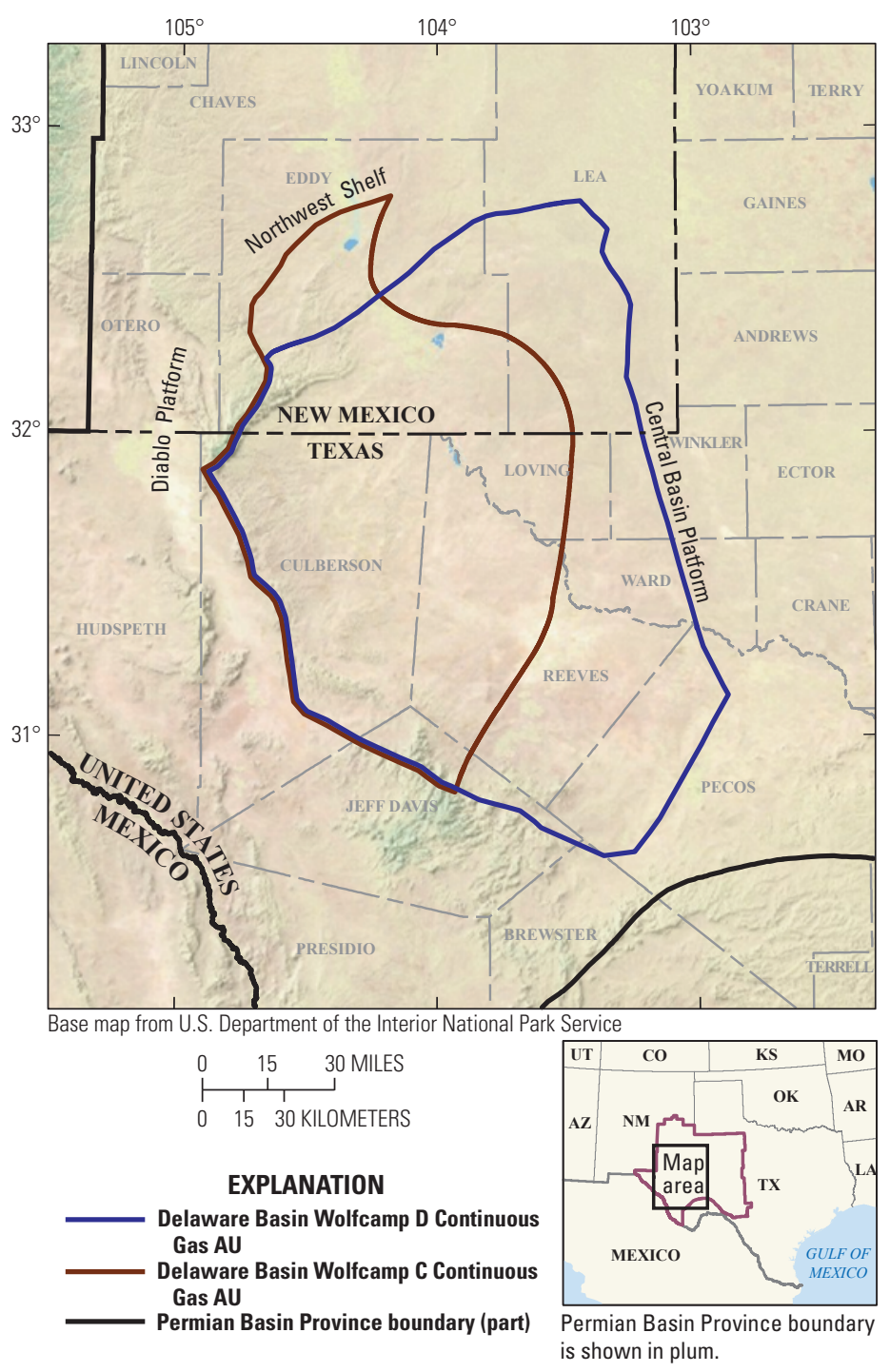

Figure 1. Map showing the Delaware Basin, Permian Basin Province, New Mexico and Texas, and the extent of the two Wolfcamp shale-gas continuous assessment units (AUs). Province boundary is from Klett and others, 1997. 


\section{Definition of Assessment Units}

Eleven continuous assessment units (AUs) were defined and quantitatively assessed in the Wolfcamp shale and the Bone Spring Formation of the Delaware Basin in the Permian Basin Province, southeast New Mexico and west Texas. Six are in the Pennsylvanian-Permian Wolfcamp shale, and five are in the Permian Bone Spring Formation. The AUs are all within the Permian Basin Paleozoic Composite Total Petroleum System (Schenk and others, 2008) and are as follows, from oldest to youngest in age: (1) Delaware Basin Wolfcamp D Continuous Gas AU, (2) Delaware Basin Wolfcamp C Continuous Gas AU, (3) Delaware Basin Wolfcamp C Continuous Oil AU, (4) Delaware Basin Wolfcamp B Lower Continuous Oil AU, (5) Delaware Basin Wolfcamp B Upper Continuous Oil AU, (6) Delaware Basin Wolfcamp A Continuous Oil AU, (7) Third Bone Spring Continuous Oil AU, (8) Second Bone Spring Continuous Oil AU, (9) First Bone Spring Continuous Oil AU, (10) Lower Avalon Shale Continuous Oil AU, and (11) Upper Avalon Shale Continuous Oil AU.

Assessment unit boundaries are constrained by the Delaware Basin boundary, as defined by Dutton and others
(2005) and Hamlin and Baumgardner (2012); extent of basinal facies; thermal maturation data (Pawlewicz and others, 2005); one-dimensional burial history modeling; isopach thickness of individual units; and structural top data (IHS Markit, 2018b). Assessment input data for the 11 AUs are summarized in table 1 .

\section{Undiscovered Resources Summary}

The USGS quantitatively assessed undiscovered, technically recoverable continuous oil and gas resources for six AUs in the Wolfcamp shale and five AUs in the Bone Spring Formation (table 2) of the Delaware Basin of the Permian Basin Province. Estimated mean resources are 46,271 million barrels of oil (MMBO), or 46.3 billion barrels of oil, with an F95-F5 range from 26,729 to 71,075 MMBO; 280,630 billion cubic feet of gas (BCFG), or 281 trillion cubic feet of associated gas, with an F95-F5 range from 108,583 to 490,074 BCFG; and 19,917 million barrels of natural gas liquids (MMBNGL) with an F95-F5 range from 7,417 to 37,349 MMBNGL (table 2).

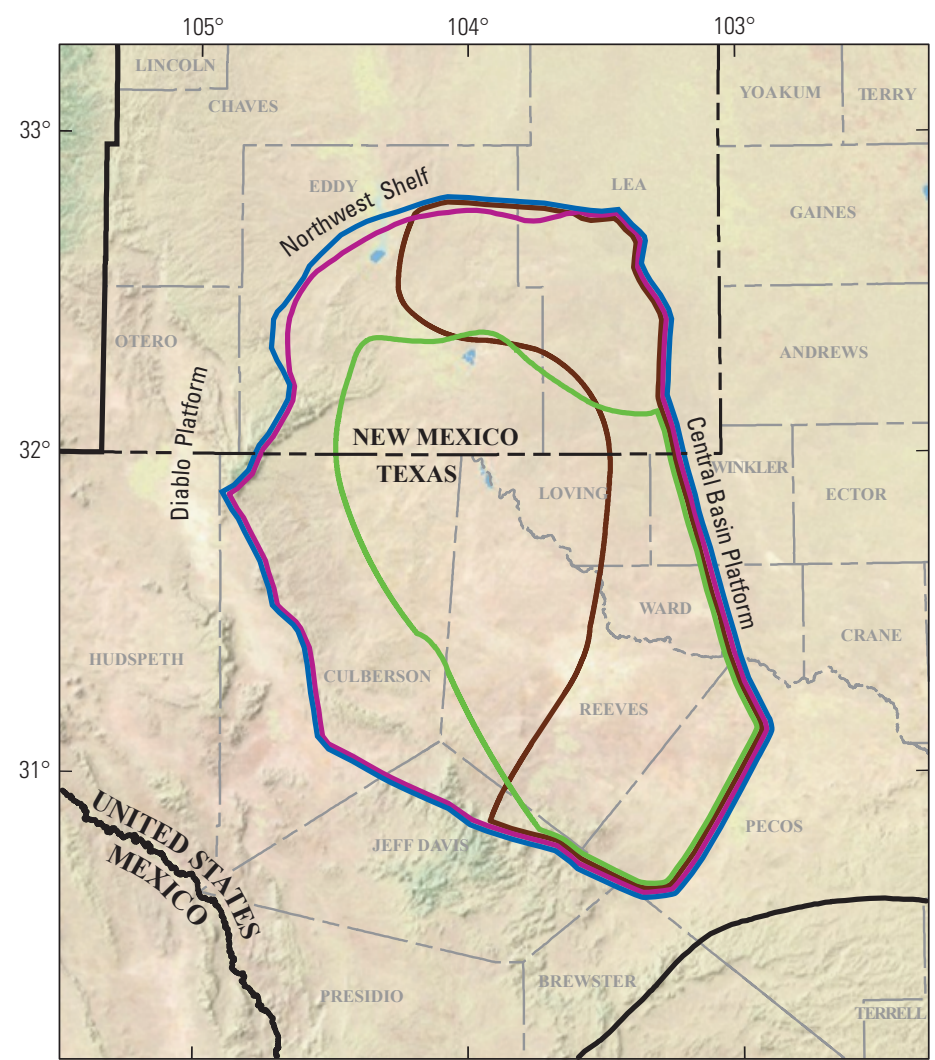

Base map from U.S. Department of the Interior National Park Service

\section{EXPLANATION}

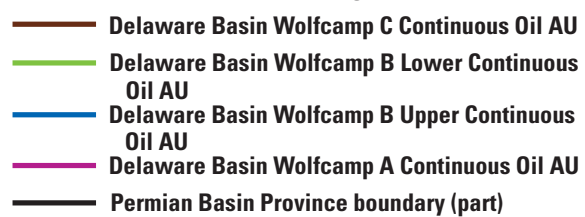

Figure 2. Map showing the Delaware Basin, Permian Basin Province, New Mexico and Texas, and the extent of the four Wolfcamp shale-oil continuous assessment units (AUs). Province boundary is from Klett and others, 1997.

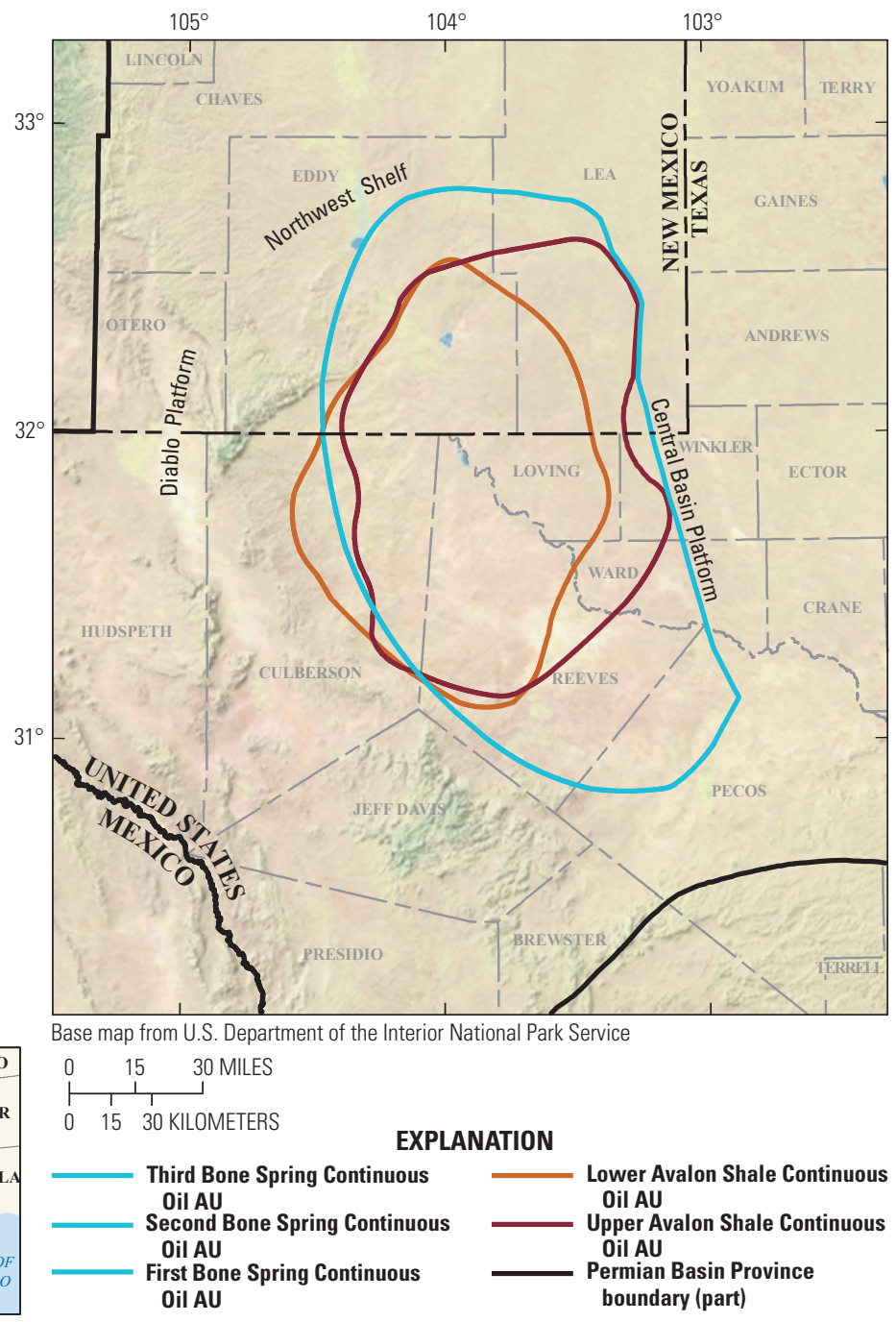

Figure 3. Map showing the Delaware Basin, Permian Basin Province, New Mexico and Texas, and the extent of the five Bone Spring Formation continuous assessment units (AUs). The Third, Second, and First Bone Spring AUs have the same extent. Province boundary is from Klett and others, 1997. 
Table 1. Key input data for 11 continuous assessment units (AUs) in the Wolfcamp shale and Bone Spring Formation of the Delaware Basin, Permian Basin Province, New Mexico and Texas.

[AU, assessment unit; \%, percent; EUR, estimated ultimate recovery per well; MMBO, million barrels of oil; BCFG, billion cubic feet of gas. Well drainage area, success ratio, and EUR are defined partly using U.S. shale-gas and shale-oil analogs. The average EUR input is the minimum, median, maximum, and calculated mean.

Shading indicates not applicable]

\begin{tabular}{|c|c|c|c|c|c|c|c|c|}
\hline \multirow{2}{*}{$\begin{array}{l}\text { Assessment input data- } \\
\text { Continuous AUs }\end{array}$} & \multicolumn{4}{|c|}{ Delaware Basin Wolfcamp D Continuous Gas AU } & \multicolumn{4}{|c|}{ Delaware Basin Wolfcamp C Continuous Gas AU } \\
\hline & Minimum & Mode & Maximum & $\begin{array}{c}\text { Calculated } \\
\text { mean }\end{array}$ & Minimum & Mode & Maximum & $\begin{array}{c}\text { Calculated } \\
\text { mean }\end{array}$ \\
\hline Potential production area of AU (acres) & 2,000 & $5,100,000$ & $7,100,000$ & $4,067,333$ & 1,500 & $3,000,000$ & $4,400,000$ & $2,467,167$ \\
\hline Average drainage area of wells (acres) & 80 & 100 & 160 & 113.3 & 80 & 100 & 160 & 113.3 \\
\hline Area untested in $\mathrm{AU}(\%)$ & 97 & 98 & 99 & 98 & 95 & 98 & 99 & 97.3 \\
\hline Success ratio $(\%)$ & 70 & 80 & 90 & 80.0 & 50 & 70 & 90 & 70 \\
\hline Average EUR (BCFG) & 1.0 & 2.5 & 4.5 & 2.579 & 1.0 & 2.5 & 4.5 & 2.579 \\
\hline AU probability & 1.0 & & & & 1.0 & & & \\
\hline \multirow{2}{*}{$\begin{array}{l}\text { Assessment input data- } \\
\text { Continuous AU }\end{array}$} & \multicolumn{4}{|c|}{ Delaware Basin Wolfcamp C Continuous Oil AU } & \multicolumn{4}{|c|}{ Delaware Basin Wolfcamp B Lower Continuous Oil AU } \\
\hline & Minimum & Mode & Maximum & $\begin{array}{c}\text { Calculated } \\
\text { mean }\end{array}$ & Minimum & Mode & Maximum & $\begin{array}{c}\text { Calculated } \\
\text { mean }\end{array}$ \\
\hline Potential production area of AU (acres) & 1,000 & $2,500,000$ & $3,300,000$ & $1,933,667$ & $1,000,000$ & $3,800,000$ & $4,500,000$ & $3,100,000$ \\
\hline Average drainage area of wells (acres) & 60 & 80 & 160 & 100 & 60 & 80 & 160 & 100 \\
\hline Area untested in $\mathrm{AU}(\%)$ & 100 & 100 & 100 & 100 & 91 & 97 & 98 & 95.3 \\
\hline Success ratio $(\%)$ & 10 & 50 & 90 & 50 & 90 & 95 & 99 & 94.7 \\
\hline Average EUR (MMBO) & 0.01 & 0.16 & 0.35 & 0.167 & 0.1 & 0.18 & 0.4 & 0.191 \\
\hline AU probability & 1.0 & & & & 1.0 & & & \\
\hline \multirow{2}{*}{$\begin{array}{l}\text { Assessment input data- } \\
\text { Continuous AU }\end{array}$} & \multicolumn{4}{|c|}{ Delaware Basin Wolfcamp B Upper Continuous Oil AU } & \multicolumn{4}{|c|}{ Delaware Basin Wolfcamp A Continuous Oil AU } \\
\hline & Minimum & Mode & Maximum & $\begin{array}{c}\text { Calculated } \\
\text { mean }\end{array}$ & Minimum & Mode & Maximum & $\begin{array}{c}\text { Calculated } \\
\text { mean }\end{array}$ \\
\hline Potential production area of AU (acres) & $1,800,000$ & $5,600,000$ & $7,750,000$ & $5,050,000$ & $4,000,000$ & $5,600,000$ & $7,600,000$ & $5,733,333$ \\
\hline Average drainage area of wells (acres) & 60 & 80 & 160 & 100 & 60 & 80 & 160 & 100 \\
\hline Area untested in $\mathrm{AU}(\%)$ & 95 & 98 & 99 & 97.3 & 92 & 95 & 96 & 94.3 \\
\hline Success ratio $(\%)$ & 90 & 95 & 99 & 94.7 & 90 & 95 & 99 & 94.7 \\
\hline Average EUR (MMBO) & 0.12 & 0.18 & 0.4 & 0.192 & 0.14 & 0.24 & 0.5 & 0.253 \\
\hline AU probability & 1.0 & & & & 1.0 & & & \\
\hline Assessment innut data- & \multicolumn{4}{|c|}{ Third Bone Spring Continuous Oil AU } & \multicolumn{4}{|c|}{ Second Bone Spring Continuous Oil AU } \\
\hline Continuous AU & Minimum & Mode & Maximum & $\begin{array}{c}\text { Calculated } \\
\text { mean }\end{array}$ & Minimum & Mode & Maximum & $\begin{array}{c}\text { Calculated } \\
\text { mean }\end{array}$ \\
\hline Potential production area of AU (acres) & $4,000,000$ & $5,000,000$ & $5,600,000$ & $4,866,667$ & $3,600,000$ & $5,000,000$ & $5,600,000$ & $4,733,333$ \\
\hline Average drainage area of wells (acres) & 120 & 160 & 180 & 153.3 & 120 & 160 & 180 & 153.3 \\
\hline Area untested in $\mathrm{AU}(\%)$ & 96 & 97 & 98 & 97 & 95 & 96 & 97 & 96 \\
\hline Success ratio $(\%)$ & 90 & 95 & 99 & 94.7 & 90 & 95 & 99 & 94.7 \\
\hline Average EUR (MMBO) & 0.14 & 0.22 & 0.45 & 0.232 & 0.1 & 0.18 & 0.3 & 0.185 \\
\hline AU probability & 1.0 & & & & 1.0 & & & \\
\hline Assessment input data- & \multicolumn{4}{|c|}{ First Bone Spring Continuous Oil AU } & \multicolumn{4}{|c|}{ Lower Avalon Shale Continuous 0il AU } \\
\hline Continuous AU & Minimum & Mode & Maximum & $\begin{array}{c}\text { Calculated } \\
\text { mean }\end{array}$ & Minimum & Mode & Maximum & $\begin{array}{c}\text { Calculated } \\
\text { mean }\end{array}$ \\
\hline Potential production area of AU (acres) & 2,000 & $3,500,000$ & $5,600,000$ & $3,034,000$ & 400,000 & $1,400,000$ & $3,000,000$ & $1,600,000$ \\
\hline Average drainage area of wells (acres) & 120 & 160 & 180 & 153.3 & 80 & 120 & 160 & 120 \\
\hline Area untested in $\mathrm{AU}(\%)$ & 97 & 98 & 99 & 98 & 91 & 95 & 97 & 94.3 \\
\hline Success ratio $(\%)$ & 70 & 80 & 90 & 80 & 70 & 80 & 90 & 80 \\
\hline Average EUR (MMBO) & 0.1 & 0.13 & 0.25 & 0.137 & 0.05 & 0.11 & 0.2 & 0.114 \\
\hline AU probability & 1.0 & & & & 1.0 & & & \\
\hline Assessment input data- & \multicolumn{4}{|c|}{ Upper Avalon Shale Continuous Oil AU } & & & & \\
\hline Continuous AU & Minimum & Mode & Maximum & $\begin{array}{c}\text { Calculated } \\
\text { mean }\end{array}$ & & & & \\
\hline Potential production area of AU (acres) & 500,000 & $2,500,000$ & $3,600,000$ & $2,200,000$ & & & & \\
\hline Average drainage area of wells (acres) & 80 & 120 & 160 & 120 & & & & \\
\hline Area untested in AU $(\%)$ & 93 & 96 & 97 & 95.3 & & & & \\
\hline Success ratio $(\%)$ & 70 & 80 & 90 & 80 & & & & \\
\hline Average EUR (MMBO) & 0.05 & 0.11 & 0.2 & 0.114 & & & & \\
\hline AU probability & 1.0 & & & & & & & \\
\hline
\end{tabular}


Table 2. Results for 11 continuous assessment units (AUs) in the Wolfcamp shale and Bone Spring Formation of the Delaware Basin, Permian Basin Province, New Mexico and Texas.

[MMBO, million barrels of oil; BCFG, billion cubic feet of gas; NGL, natural gas liquids; MMBNGL, million barrels of natural gas liquids. Results shown are fully risked estimates. F95 represents a 95-percent chance of at least the amount tabulated; other fractiles are defined similarly. Fractiles are additive under the assumption of perfect positive correlation. Shading indicates not applicable]

\begin{tabular}{|c|c|c|c|c|c|c|c|c|c|c|c|c|c|c|}
\hline \multirow{3}{*}{$\begin{array}{l}\text { Total petroleum system } \\
\text { and assessment units (AUs) }\end{array}$} & \multirow{3}{*}{$\begin{array}{c}\text { AU } \\
\text { prob- } \\
\text { ability }\end{array}$} & \multirow{3}{*}{$\begin{array}{c}\text { Accu- } \\
\text { mulation } \\
\text { type }\end{array}$} & \multicolumn{12}{|c|}{ Total undiscovered resources } \\
\hline & & & \multicolumn{4}{|c|}{ Oil (MMBO) } & \multicolumn{4}{|c|}{ Gas (BCFG) } & \multicolumn{4}{|c|}{ NGL (MMBNGL) } \\
\hline & & & F95 & F50 & F5 & Mean & F95 & F50 & F5 & Mean & F95 & F50 & F5 & Mean \\
\hline \multicolumn{15}{|c|}{ Permian Basin Paleozoic Composite Total Petroleum System } \\
\hline $\begin{array}{l}\text { Delaware Basin Wolfcamp D Continuous } \\
\text { Gas AU }\end{array}$ & 1.0 & Gas & & & & & 22,718 & 72,821 & 124,966 & 72,920 & 850 & 2,794 & 5,405 & 2,917 \\
\hline $\begin{array}{l}\text { Delaware Basin Wolfcamp C Continuous } \\
\text { Gas AU }\end{array}$ & 1.0 & Gas & & & & & 11,757 & 37,793 & 67,845 & 38,510 & 438 & 1,455 & 2,924 & 1,539 \\
\hline $\begin{array}{l}\text { Delaware Basin Wolfcamp C Continuous } \\
\text { Oil AU }\end{array}$ & 1.0 & Oil & 382 & 1,467 & 3,477 & 1,635 & 5,585 & 21,685 & 52,927 & 24,486 & 404 & 1,667 & 4,517 & 1,961 \\
\hline $\begin{array}{l}\text { Delaware Basin Wolfcamp B Lower } \\
\text { Continuous Oil AU }\end{array}$ & 1.0 & Oil & 2,642 & 5,297 & 8,838 & 5,458 & 9,519 & 20,616 & 38,280 & 21,815 & 869 & 2,011 & 4,078 & 2,181 \\
\hline $\begin{array}{l}\text { Delaware Basin Wolfcamp B Upper } \\
\text { Continuous Oil AU }\end{array}$ & 1.0 & Oil & 4,606 & 8,861 & 14,657 & 9,154 & 16,524 & 34,608 & 63,189 & 36,601 & 1,486 & 3,382 & 6,799 & 3,661 \\
\hline $\begin{array}{l}\text { Delaware Basin Wolfcamp A Continuous } \\
\text { Oil AU }\end{array}$ & 1.0 & Oil & 8,406 & 12,791 & 19,556 & 13,229 & 14,297 & 25,219 & 42,988 & 26,492 & 1,259 & 2,476 & 4,626 & 2,648 \\
\hline Third Bone Spring Continuous Oil AU & 1.0 & Oil & 4,941 & 6,468 & 9,521 & 6,739 & 7,906 & 12,975 & 20,851 & 13,485 & 501 & 896 & 1,551 & 943 \\
\hline Second Bone Spring Continuous Oil AU & 1.0 & Oil & 3,869 & 5,096 & 6,878 & 5,191 & 6,201 & 10,120 & 15,435 & 10,370 & 391 & 696 & 1,158 & 725 \\
\hline First Bone Spring Continuous Oil AU & 1.0 & Oil & 658 & 2,113 & 3,588 & 2,113 & 2,492 & 8,119 & 15,564 & 8,451 & 165 & 555 & 1,147 & 592 \\
\hline Lower Avalon Shale Continuous Oil AU & 1.0 & Oil & 507 & 1,096 & 1,984 & 1,153 & 4,810 & 10,790 & 20,864 & 11,527 & 440 & 1,053 & 2,210 & 1,151 \\
\hline Upper Avalon Shale Continuous Oil AU & 1.0 & Oil & 718 & 1,573 & 2,576 & 1,599 & 6,774 & 15,379 & 27,165 & 15,973 & 614 & 1,496 & 2,934 & 1,599 \\
\hline Total undiscovered continuous resources & & & 26,729 & 44,762 & 71,075 & 46,271 & 108,583 & 270,125 & 490,074 & 280,630 & 7,417 & 18,481 & 37,349 & 19,917 \\
\hline
\end{tabular}

\section{References Cited}

Dutton, S.P., Kim, E.M., Broadhead, R.F., Raatz, W.D., Breton, C.L., Ruppel, S.C., and Kerans, C., 2005, Play analysis and leading-edge oil-reservoir development methods in the Permian basin-Increased recovery through advanced technologies: American Association of Petroleum Geologists Bulletin, v. 89, no. 5, p. 553-576.

Gaswirth, S.B., Marra, K.R., Lillis, P.G., Mercier, T.J., LeathersMiller, H.M., Schenk, C.J., Klett, T.R., Le, P.A., Tennyson, M.E., Hawkins, S.J., Brownfield, M.E., Pitman, J.K., and Finn, T.M., 2016, Assessment of undiscovered continuous oil resources in the Wolfcamp shale of the Midland Basin, Permian Basin Province, Texas, 2016: U.S. Geological Survey Fact Sheet 2016-3092, 4 p.

Hamlin, H.S., and Baumgardner, R.W., 2012, Wolfberry (Wolfcampian-Leonardian) deep-water depositional systems in the Midland Basin-Stratigraphy, lithofacies, reservoirs, and source rocks: The University of Texas at Austin, Bureau of Economic Geology, Report of Investigations No. 277, 61 p.

IHS Markit $^{\circledR}, 2018 \mathrm{a}$, Enerdeq ${ }^{\mathrm{TM}}$ US well history and production database: Englewood, Colo., IHS Markit, accessed April 2018 at http://www.ihsenergy.com. [Available from IHS Markit, 15 Inverness Way East, Englewood, CO 80112.]
IHS Markit ${ }^{\text {B }}, 2018 b$, PRODFit ${ }^{\text {TM }}$ database: Englewood, Colo., IHS Markit, accessed May 2018 at http://www.ihsenergy.com. [Available from IHS Markit, 15 Inverness Way East, Englewood, CO 80112.]

Klett, T.R., Ahlbrandt, T.S., Schmoker, J.W., and Dolton, G.L., 1997, Ranking of the world's oil and gas provinces by known petroleum volumes: U.S. Geological Survey Open-File Report 97-463, 1 CD-ROM. [Also available at https://pubs.usgs.gov/of/1997/ ofr-97-463/97463.html.]

Marra, K.R., Gaswirth, S.B., Schenk, C.J., Leathers-Miller, H.M., Klett, T.R., Mercier, T.J., Le, P.A., Tennyson, M.E., Finn, T.M., Hawkins, S.J., and Brownfield, M.E., 2017, Assessment of undiscovered oil and gas resources in the Spraberry Formation of the Midland Basin, Permian Basin Province, Texas, 2017: U.S. Geological Survey Fact Sheet 2017-3029, 2 p.

Pawlewicz, M., Barker, C.E., and McDonald, S., 2005, Vitrinite reflectance data for the Permian Basin, west Texas and southeast New Mexico: U.S. Geological Survey Open-File Report 2005-1171, 25 p.

Schenk, C.J., Pollastro, R.M., Cook, T.A., Pawlewicz, M.J., Klett, T.R., Charpentier, R.R., and Cook, H.E., 2008, Assessment of undiscovered oil and gas resources of the Permian Basin Province of west Texas and southeast New Mexico, 2007: U.S. Geological Survey Fact Sheet 2007-3115, 4 p.

\section{For More Information}

Assessment results are also available at the USGS Energy Resources Program website at https://energy.usgs.gov.

\section{Delaware Basin Continuous Resources Assessment Team}

Stephanie B. Gaswirth, Katherine L. French, Janet K. Pitman, Kristen R. Marra, Tracey J. Mercier, Heidi M. Leathers-Miller, Christopher J. Schenk, Marilyn E. Tennyson, Cheryl A. Woodall, Michael E. Brownfield, Thomas M. Finn, and Phuong A. Le 